\title{
ТРАНСФОРМАЦИЯ СОЦИАЛЬНЫХ ПРАКТИК ОТВЕТСТВЕННОГО ОТЦОВСТВА В ДЕВИАНТНЫЕ ФОРМЫ СОЦИАЛЬНОЙ АКТИВНОСТИ
}

\author{
Карпова Анна Юрьевна, \\ belts@tpu.ru \\ Савельев Алексей Олегович, \\ sava@tpu.ru \\ Кузнецов Сергей Анатольевич, \\ ksa11@tpu.ru
}

Национальный исследовательский Томский политехнический университет, Россия, 634050, г. Томск, пр. Ленина, 30

Карпова Анна Юрьевна, доктор социологических наук, профессор отделения социальногуманитарных наук Школы базовой инженерной подготовки Национального исследовательского Томского политехнического университета.

Савельев Алексей Олегович, кандидат технических наук, доцент отделения информационных технологий Школы базовой инженерной подготовки Национального исследовательского Томского политехнического университета.

Кузнецов Сергей Анатольевич, инженер отделения информационных технологий Школы базовой инженерной подготовки Национального исследовательского Томского политехнического университета.

Изучение социальных практикответственного отцовства актуализируется в настоящее время в контексте трансформации института отцовства. Особое внимание исследователей направлено на социальное конструирование ответственного отцовства в медиапространстве (профильные сообщества в социальных сетях). Проблема в том, что внутри отцовских сообществ в социальных сетях появляются маркеры радикальныхмнений, идей, которые по сути являются предиктором перехода к девиантным формам социальной активности и деструктивным практикам. Цель оценка семантического сходства и перекрестных связей между сообществами «Союза отцов» и «Совета отцов» и сообществами с признаками радикализации. Методы: контент-анализ, web тіпing. По результатам исследования обозначено наличие устойчивой связи тематического и семантического сходства между отдельными сообществами «отцов» и «пролайферов». Отмечаются признаки трансформации социальных практикответственного отцовства в девиантные формы социальной активности. Подчеркивается, что применение разнообразных методов web mining повышает возможность оценить влияние радикальных мнений и идей в онлайн-среде, соответственно, возрастает возможность предупреждения и предотвращения такой формы социальной активности в сообществах ответственныхотцов, какраспространение радикальной идеологии. Также обозначены направления будущих исследований.

Ключевыеслова: Генеративное поведение, радикализация, экстремизм, сетевая топология, big data, web mining.

\section{Введение}

Изучение социальных практик ответственного отцовства в российском исследовательском поле в настоящее время актуализировано в контексте исследования трансформации института отцовства. Как подчеркивает один из руководителей данного направления Ч.И. Ильдарханова, «фокус научных исследований все больше смещается 
к важному, но малоизученному объекту: феномену отцовства в рамках генеративного поведения мужчин» $[1,2]$. Сам термин «генеративное поведение» еще не получил в исследовательской среде так называемого академического консенсусного определения и рассматривается как с точки зрения понятия, расширяющего термин «репродуктивное поведение», так и с точки зрения контекстуальной интерпретации генеративности как необходимости изучать данный феномен в широком формате демографического, репродуктивного, прокреативного поведения, а также с позиций деятельностного подхода, не ограничиваясь какими-либо рамками [3]. Наиболее перспективным представляется подход Л.А. Давлетшиной, в котором автор предлагает сосредоточить внимание «на выявлении и обобщении базовых характеристик, а также тенденций трансформации моделей генеративного поведения российских мужчин в условиях преобразования института семьи... детального анализа не только структуры генеративного поведения и факторов, влияющих на него, но и комплексного анализа влияния различных моделей генеративного поведения российских мужчин на формирование повседневных практик мужского родительства» [4, с. 54]. Генеративность, с точки зрения контекстуального определения, следует рассматривать:

- как совокупность действий в процессе воспроизводства «новых поколений, формирование зрелых в социальном и психофизиологическом отношении личностей... но и социализацию как совокупность действий и отношений, обеспечивающие усвоение ребёнком системы норм и ценностей, которые дают возможность ему функционировать как полноправному члену общества» [5, с. 25];

- как деятельность «в аспекте генерации человеческого и гражданского капитала» [6, с. 239], подразумевающую осознанное, ответственное поведение как в формировании семейных отношений и ценностей, так и в формате ответственного проявления в публичной сфере гражданской позиции, политического участия и других форм позитивных социальных практик [7, 8];

- в контексте концепции гибридного отцовства, в которой, по мнению зарубежных авторов, включаются стереотипно женские стили, например, эмоциональная выразительность, патриархат, дифференцируются доминирующие и подчиненные формы мужественности, относящиеся к мужскому родительству [9];

- в контексте изучения норм отцовства, которые претерпевают трансформации «по мере того, как поддержка гендерного разделения труда теряет свои позиции, переход к отцовству становится все более благоприятным для удовлетворения жизнью для обоих полов» $[10$, с. 54].

В изучении генеративного поведения российских мужчин основной фокус направлен на обследование репродуктивных стратегий, родительских практик отцовства «в различных проявлениях: участии и вовлеченности в процесс первичного ухода за детьми, воспитания, перераспределения домашних обязанностей и т. д.» [1, с. 211]. Российские исследователи рассматривают в данном контексте предпосылки и ограничения трансформации современного института отцовства [11]. Но при этом недостаточно внимания уделяется социальному конструированию ответственного отцовства в медиапространстве - профильных сообществ «Союз отцов» и «Совет отцов» (общественные объединения) в социальных сетях. При том, что роль социальных медиа в формировании общественного восприятия феномена ответственного отцовства, представляющего собой форму гражданского активизма, является важным фактором формирования моделей генеративного поведения российских мужчин. Проблема, которую необходимо решить, заключается в противоречии между интерпретацией самими мужчинами (членами сообщества) идентификационных маркеров, размещаемых на интер- 
нет-платформах сообществ ответственных отцов, которые призваны транслировать ценности родительства, семейно-детского образа жизни, и непосредственно социальной идентификацией этих символов потребителями контента этих сайтов (не членами сообщества). Парадокс заключается в том, что внутри отцовских сообществ присутствуют маркеры радикальных мнений, идей, которые по сути являются предиктором перехода к девиантным формам социальной активности и деструктивным практикам. В контексте обозначенной проблемы цель исследования - это оценка семантического сходства и перекрестных связей между сообществами «Союза отцов» и «Совета отцов» и сообществами с признаками радикализации. Структура статьи состоит из блоков, каждый из которых раскрывает решение одной из задач исследования. В первой части работы мы сосредоточились на описании выборки, методах и инструментах исследования. Во второй части работы мы изложили основные эмпирические данные. В этой части работы мы описываем отличительные особенности семантического сходства на основе применения методов машинного обучения и статистических мер значимости слов в постах. В заключении обозначены перспективы дальнейшего направления исследований и предпочтительный вариант исследовательской стратегии для изучения онлайнрадикализации в профильных сообществах «Союз отцов» и «Совет отцов».

\section{Материалы и методы}

Выборка исследования - 55 профильных сообществ «Союз отцов», «Совет отцов». Для изучения перекрестных связей и меры семантического сходства были выбраны 13 сообществ «пролайферов» социальной сети «ВКонтакте» [12]. Пролайферы (от англ. pro-life - в защиту жизни [13]) - это общественное движение, целью которого является запрет абортов. Несмотря на декларируемые гуманные цели, с позиций защиты традиционных семейных ценностей, здоровья женщин, ценности и права на жизнь сторонники этого движения проявляют крайне радикальные взгляды, отказывая женщинам в праве на самостоятельное решение, зачастую используют заведомо ложную информацию или не подтвержденную исследованиями информацию о влиянии аборта на здоровье женщин, искажают данные официальной статистики $[14,15]$. Кроме того, сторонники данного движения на западе уже отмечены применением деструктивных, противоправных практик, например угрозы и реальные расправы над врачами, женщинами, нападения на клиники [16], доксинг (doxxing) ${ }^{1}$ - онлайн-преследование с публикацией конфиденциальной, персональной информации в Интернете [17]. Сообщества, противопоставляющие себя пролайферам, получили название «за выбор» (pro-choice за право выбора) [18]. Они считают, что у каждого человека есть основное право решать, когда и надо ли иметь детей. Эти сообщества так же активны в социальных медиа, как и пролайферы, и ведут не менее агрессивную пропаганду своей позиции [19]. В российских социальных медиа представлены обе позиции, но активность пролайферов значительно выше, поэтому для изучения семантического сходства были выбраны сообщества пролайферов.

В результате собрана база данных, включающая доступную из открытых аккаунтов статистику сообществ. Информация собиралась через API на открытых данных тематических сообществ, аккаунтов социальной сети «ВКонтакте» с использованием модуля программного алгоритма, разработанного проектной группой ТПУ. Сбор персональных данных, или данных, позволяющих идентифицировать пользователя, не осуществлялся. Программный модуль включает несколько функций, созданных для авто-

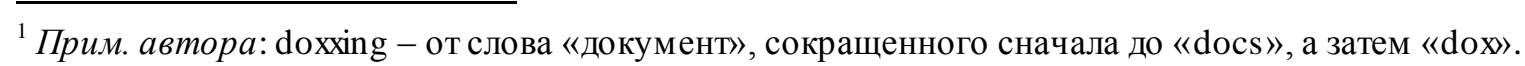


матического поиска, обработки и анализа больших данных социальных сетей. Модуль разработан первоначально для изучения сообществ ультраправого толка в российской социальной сети «ВКонтакте», протестирован в экспериментальных исследованиях под разные кейсы. Это инструмент для сопровождения научных исследований в сфере общественно-политических наук по изучению процесса онлайн-радикализации молодежи в социальных сетях, который проектировался как часть многофункционального средства [20]. В настоящий момент тестируются новые функции программного модуля. Для целей оценки степени взаимного сходства отдельных групп сообществ социальных медиа использованы три основные метрики: общность подписчиков, тематическое сходство и семантическое сходство (с учетом контекста) [21]. Тематическое сходство определяется через меру статистической значимости слов TF-IDF. Применяется относительное значение - отношение количества упоминаний нужного термина к общему количеству слов в тексте. IDF (inverse document frequency) - инверсия частоты, с которой некоторое слово встречается в документах коллекции. Семантическое сходство выражено косинусным расстоянием между векторизованным методом doc2vec текстовым и контентом рассматриваемых сообществ. Doc2vec является расширением подхода word2vec, который базируется на распределенном представлении слов, изученных нейронными сетями, но при этом общая логика работы применяется к уровню документа, а не слова для оценки сходства сообществ на основе содержания публикуемых сообщений.

Были поставлены следующие задачи: собрать статистику по половозрастным признакам сообществ «Союз отцов», «Совет отцов»; статистику по постам за год, вычислить среднее количество постов в день; данные по геолокации; составить топ наиболее активных сообществ по городам России; произвести оценку семантического сходства между сообществами «Союз отцов», «Совет отцов» и «пролайферов».

\section{Статистические данные}

В таблице приведена краткая статистическая информация о рассматриваемых в рамках настоящей работы сообществах социальной сети «ВКонтакте», аффилированных с сообществами «Союз отцов» и «Совет отцов». Фоновый цветовой градиент в ячейках таблицы от зеленого к красному используется для отображения положения значения по шкале от максимального к минимальному соответственно. Отдельного комментария заслуживает колонка «Пол». В социальной сети «ВКонтакте» в профиле пользователя это значение не может быть пустым. По умолчанию, при регистрации новой учетной записи пользователя, значение поля «Пол» указано как «мужской». Таким образом, преобладание «мужских» учетных записей среди подписчиков сообществ не может быть отнесено к значимым характеристикам, поскольку может отражать факт наличия большого количества подписчиков, которые при создании учетной записи «согласились» со значением, предлагаемым по умолчанию. При этом обратная ситуация преобладание «женских» учетных записей среди подписчиков - потенциально является значимым фактором, требующим дополнительного рассмотрения. Также можно отметить, что большее число подписчиков не всегда коррелирует с большей же активностью в рамках сообщества. Помимо этого, при отсутствии дополнительных способов верификации информации, указываемой пользователями в своих учетных записях, данные табл. 1 не могут быть оценены с точки зрения репрезентативности.

На рис. 1 в виде «облака слов» представлена визуализация 30 наиболее значимых, согласно метрике TF-IDF, слов для сообществ «отцов» (рис. 1, a) и «пролайферов» (рис. 1, б). 
Таблица. Статистические данные сообществ «Союз отцов» и «Совет отцов» Table. Statistical data from the Fathers' Union and Fathers' Council communities

\begin{tabular}{|c|c|c|c|c|c|c|c|}
\hline \multirow{2}{*}{$\begin{array}{c}\text { Подписчики } \\
\text { Followers }\end{array}$} & \multicolumn{3}{|c|}{ Возраст/Age } & \multicolumn{2}{|c|}{ Пол/Gender, \% } & \multicolumn{2}{|c|}{$\begin{array}{l}\text { Активность, кол-во постов } \\
\text { Activity, number of posts }\end{array}$} \\
\hline & $\begin{array}{l}\text { Медиана } \\
\text { Median }\end{array}$ & $\begin{array}{c}\text { Средний } \\
\text { Average }\end{array}$ & $\begin{array}{l}\text { Не указан, \% } \\
\text { Not specified }\end{array}$ & $\begin{array}{c}\text { муж. } \\
\text { masculine }\end{array}$ & $\begin{array}{l}\text { жен. } \\
\text { female }\end{array}$ & $\begin{array}{c}\text { За год } \\
\text { In a year }\end{array}$ & $\begin{array}{l}\text { Среднее за } 1 \text { день } \\
\text { Average in a day }\end{array}$ \\
\hline 103 & 40,5 & 41,31 & 47,57 & 81,55 & 18,45 & 44 & 0,12 \\
\hline 99 & 40 & 37,37 & 69,7 & 45,45 & 54,55 & 2 & 0,01 \\
\hline 263 & 38 & 39,88 & 59,7 & 44,87 & 55,13 & 246 & 0,66 \\
\hline 982 & 35 & 35,03 & 54,89 & 40,73 & 59,27 & 463 & 1,24 \\
\hline 796 & 37 & 38,55 & 62,06 & 36,43 & 63,57 & 14 & 0,04 \\
\hline 2101 & 39 & 40,35 & 61,16 & 71,39 & 28,61 & 332 & 0,89 \\
\hline 106 & 39 & 39,51 & 51,89 & 70,75 & 29,25 & 7 & 0,02 \\
\hline 30 & 38 & 38,59 & 43,33 & 83,33 & 16,67 & 1 & 0 \\
\hline 18 & 34 & 37 & 72,22 & 55,56 & 44,44 & 1 & 0 \\
\hline 300 & 37 & 37,25 & 61 & 37,67 & 62,33 & 66 & 0,18 \\
\hline 1838 & 38 & 38,82 & 51,25 & 60,39 & 39,61 & 116 & 0,31 \\
\hline 567 & 40 & 41,04 & 66,49 & 44,44 & 55,56 & 88 & 0,24 \\
\hline 1146 & 32 & 33,57 & 40,75 & 59,86 & 40,14 & 85 & 0,23 \\
\hline 1732 & 33 & 34,47 & 61,55 & 52,54 & 47,46 & 59 & 0,16 \\
\hline 1135 & 38 & 40,37 & 51,98 & 83,52 & 16,48 & 8 & 0,02 \\
\hline 810 & 34 & 35,27 & 59,38 & 50,74 & 49,26 & 6 & 0,02 \\
\hline 419 & 40,5 & 43,26 & 51,31 & 79,47 & 20,53 & 23 & 0,06 \\
\hline 772 & 38 & 38,95 & 63,86 & 26,55 & 73,45 & 150 & 0,4 \\
\hline 818 & 37 & 37,75 & 57,33 & 69,68 & 30,32 & 286 & 0,77 \\
\hline 1058 & 38 & 39,88 & 62,1 & 53,02 & 46,98 & 130 & 0,35 \\
\hline 555 & 39 & 39,41 & 63,42 & 54,59 & 45,41 & 44 & 0,12 \\
\hline 1280 & 39 & 40,32 & 58,91 & 59,61 & 40,39 & 250 & 0,67 \\
\hline 291 & 36 & 37,93 & 62,2 & 58,76 & 41,24 & 1 & 0 \\
\hline 317 & 34 & 35,62 & 64,67 & 42,90 & 57,1 & 1 & 0 \\
\hline 272 & 37,5 & 38,75 & 66,18 & 11,40 & 88,6 & 1 & 0 \\
\hline 253 & 37 & 38,38 & 64,03 & 50,59 & 49,41 & 1 & 0 \\
\hline 311 & 34 & 34,37 & 54,98 & 27,33 & 72,67 & 61 & 0,16 \\
\hline 187 & 39 & 38,86 & 58,29 & 63,10 & 36,9 & 30 & 0,08 \\
\hline 221 & 37 & 37,59 & 57,01 & 39,82 & 60,18 & 11 & 0,03 \\
\hline 360 & 38 & 38,55 & 58,06 & 32,50 & 67,5 & 107 & 0,29 \\
\hline 160 & 36,5 & 36,24 & 63,75 & 37,50 & 62,5 & 3 & 0,01 \\
\hline 147 & 34 & 36,65 & 62,59 & 38,10 & 61,9 & 1 & 0 \\
\hline 148 & 39 & 39,65 & 51,35 & 64,86 & 35,14 & 23 & 0,06 \\
\hline 188 & 38 & 42,97 & 63,83 & 43,62 & 56,38 & 18 & 0,05 \\
\hline 139 & 39 & 40,15 & 56,83 & 54,68 & 45,32 & 80 & 0,21 \\
\hline 186 & 36 & 37,19 & 68,28 & 41,94 & 58,06 & 17 & 0,05 \\
\hline 107 & 36,5 & 36,8 & 58,88 & 50,47 & 49,53 & 4 & 0,01 \\
\hline 535 & 37 & 39,65 & 48,22 & 50,47 & 49,53 & 534 & 1,43 \\
\hline 159 & 38,5 & 40,56 & 57,23 & 50,31 & 49,69 & 44 & 0,12 \\
\hline 177 & 36 & 38,29 & 46,89 & 75,71 & 24,29 & 32 & 0,09 \\
\hline 145 & 35 & 38,88 & 46,21 & 33,10 & 66,9 & 28 & 0,08 \\
\hline 379 & 33 & 33,51 & 56,73 & 26,39 & 73,61 & 78 & 0,21 \\
\hline 1195 & 31 & 32,59 & 42,93 & 57,15 & 42,85 & 14 & 0,04 \\
\hline 421 & 37 & 37,6 & 64,61 & 35,39 & 64,61 & 115 & 0,31 \\
\hline 116 & 36 & 38,48 & 63,79 & 48,28 & 51,72 & 90 & 0,24 \\
\hline 187 & 36 & 37,84 & 60,43 & 45,99 & 54,01 & 11 & 0,03 \\
\hline 157 & $\overline{42}$ & 40,95 & 58,6 & 49,68 & 50,32 & 54 & 0,14 \\
\hline 146 & 39,5 & 41,94 & 56,16 & 35,62 & 64,38 & 59 & 0,16 \\
\hline 95 & 37 & 38,9 & 67,37 & 81,05 & 18,95 & 18 & 0,05 \\
\hline 104 & 41 & 39,16 & 64,42 & 46,15 & 53,85 & 28 & 0,07 \\
\hline 389 & 32 & 32,59 & 67,87 & 50,13 & 49,87 & 61 & 0,16 \\
\hline 502 & 23 & 26,23 & 38,05 & 61,55 & 38,45 & 32 & 0,09 \\
\hline 167 & 37 & 37,51 & 52,69 & 49,10 & 50,9 & 29 & 0,08 \\
\hline 2147 & 26 & 28,18 & 80,2 & 22,96 & 77,04 & 50 & 0,13 \\
\hline 35 & 39 & 39,65 & 51,43 & 48,57 & 51,43 & 107 & 0,29 \\
\hline
\end{tabular}



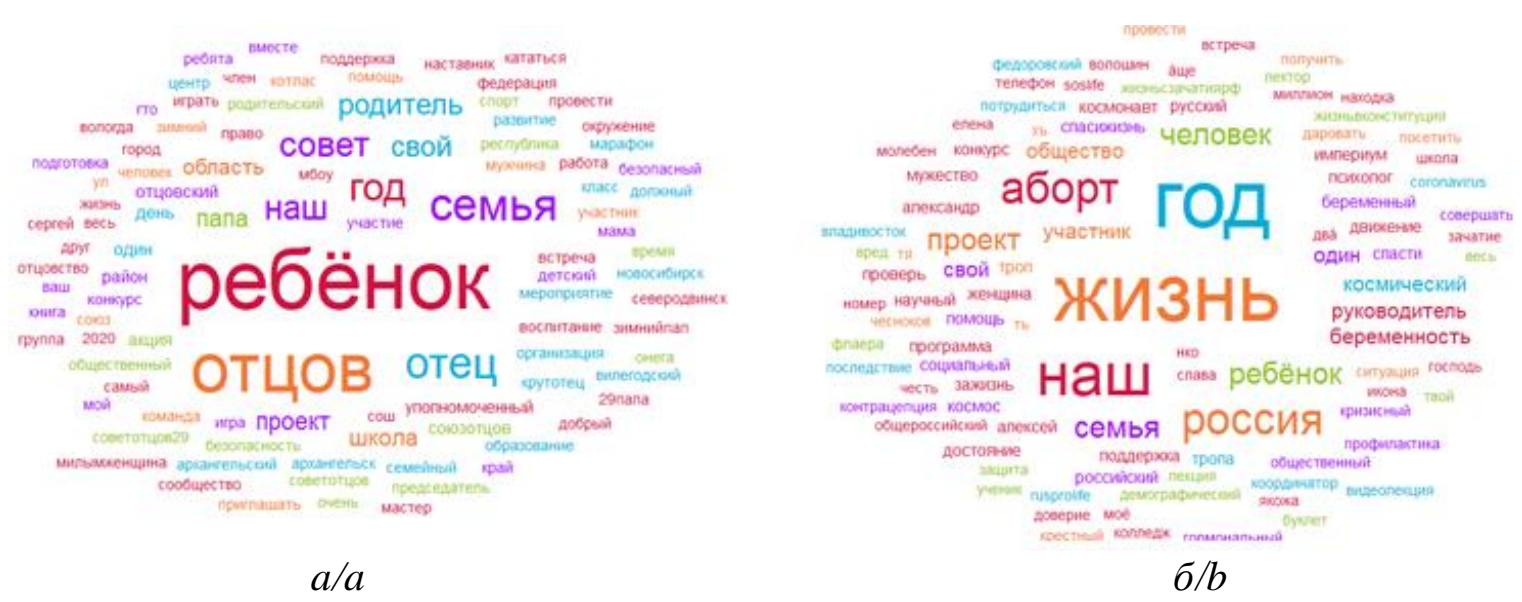

Рис. 1. Облако значимых слов рассматриваемых сообществ:

а) слова для сообществ «отцов»; б) слова для сообществ «пролайферов»

Fig. 1. Cloud of meaningful words of the communities:

a) words for communities of «fathers»; b) words for community of «pro-lifers»

\section{Сетевые топологии связей рассматриваемых сообществ}

Для оценки связей между рассматриваемыми сообществами были использованы выше обозначенные метрики: общность подписчиков, тематическое сходство и семантическое сходство. На рис. 2 приведена визуализация фрагментов сетевых топологий в виде ненаправленного графа. Узлы представляют собой рассматриваемые сообщества в социальной сети «ВКонтакте». Вертикальной штриховкой выделены сообщества «отцов», сплошной штриховкой - «пролайферов». Ребра между узлами отражают наличие связи согласно метрикам. Узлы, не связанные ребрами, исключены. Рис. 2, $a$, отражает топологию «общие подписчики», рис. $2, \sigma, 6,-$ «тематическое сходство» и «семантическое сходство» соответственно.

Приведенная визуализация позволяет сформулировать следующие выводы:

1. Рассматриваемые классы сообществ не имеют значимых взаимосвязей по метрике «общие подписчики». При этом класс «отцов» представляет собой плотный кластер, в то время как класс «пролайферов» представлен двумя, сравнительно небольшими кластерами.

2. Тематическое сходство демонстрирует наличие связей ряда сообществ «пролайферов» с одним сообществом «отцов», являющимся частью обширного кластера и занимающим одно из центральных мест в нем.

3. Использование метрики «семантическое сходство» позволило выявить наличие устойчивой связи между отдельными сообществами «отцов» и «пролайферов», что означает наличие не только общей тематики в публикуемом текстовом контенте, но также и сходство контекстов относительно одних и тех же значимых слов.

Визуализация позволила интерпретировать связи сообществ, а также оценить значимость через вычисление степеней близости, посредничества и влияния. Полученная информация в дальнейшем может быть использована при моделировании процессов информационной диффузии в рамках сообществ. Фактически в сообществах «Союз отцов» и «Совет отцов» имеются признаки трансформации социальных практик ответственного отцовства в девиантные формы социальной активности через: 1) усиление агрессивной рит орики и рост женоненавистнического дискурса; 2) укрепление связей посредством социальных сетей между сообществами с признаками деструктивного контента и сообществами ответственных отцов, которые становятся шлюзом для радикализации. 

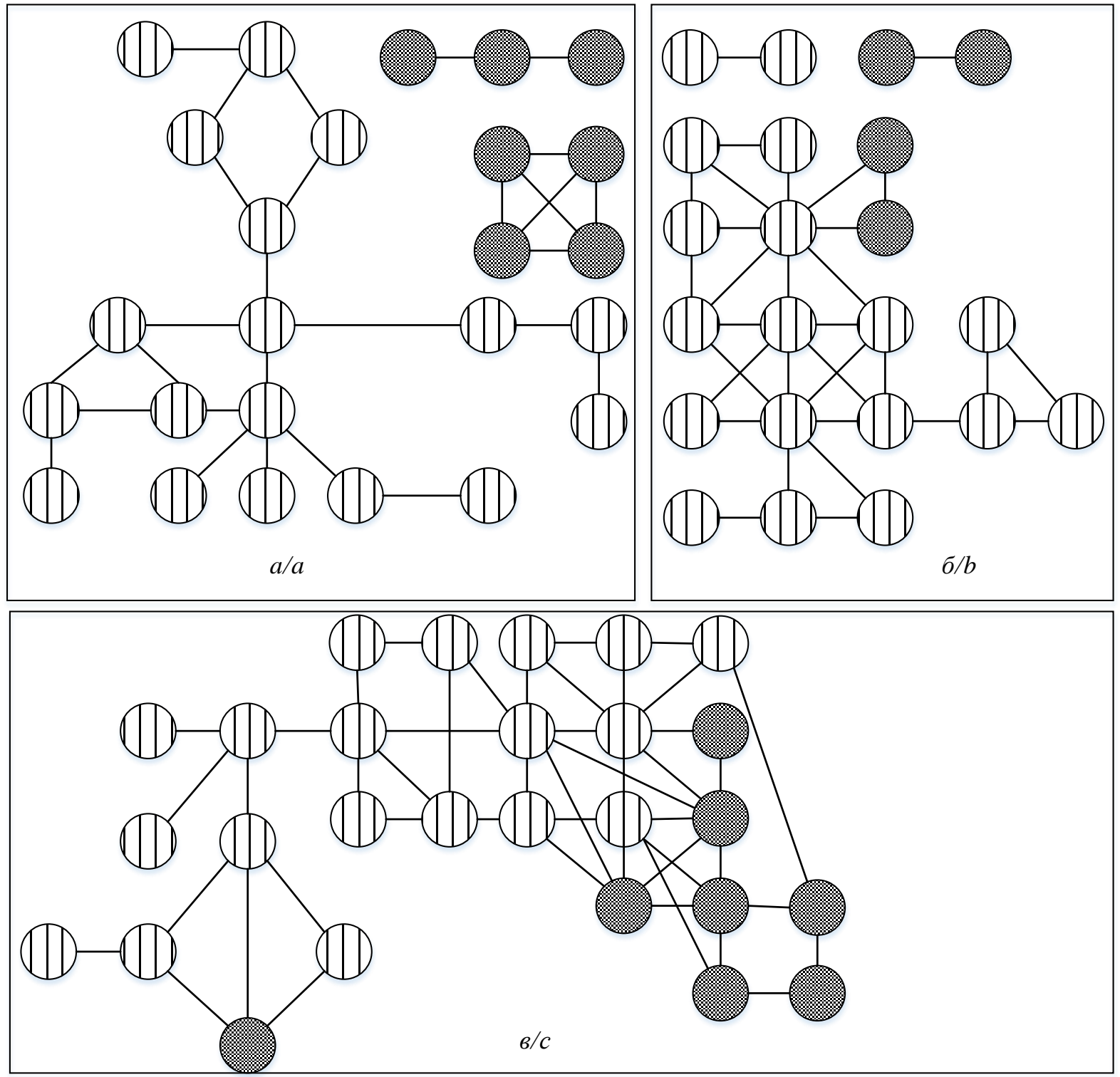

Pис. 2. Фрагменты сетевых топологий связей сообществ «отцов» (вертикальная штриховка) и «пролайферов» (сплошная штриховка): а) топология «общие подписчики»; б) топология «тематическое сходство»;

в) топология "семантическое сходство»

Fig. 2. Fragments of the network topologies of the "fathers» (vertical shading) and «pro-lifers» (solid shading): a) topology "common subscribers»; b) topology «thematic similarity»; c) topology «semantic similarity»

\section{Заключение}

Социальные отношения в онлайн-среде оказывают значительное влияние на путь радикализации. Сам феномен радикальной парадигмы в отцовской среде крайне негативно влияет как на социализацию молодежи, отцами которой являются и мужчины, практикующие мировоззрение, представляющее собой определенную форму девиации, так и на восприятие отцовства общественностью. Практической значимостью исследования является возможность предупредить и предотвратить такую форму социальной активности в сообществах ответственных отцов, как распространение радикальной 
идеологии. Учитывая динамичный характер экстремистских организаций, именно применение исследователями разнообразных методов web mining повышает возможность оценить влияние радикальных идеологий в онлайн-среде. Результаты изучения семантического сходства между сообществами «Союза отцов» и «Совета отцов» и сообществами с признаками радикализации позволили выделить направления будущих исследований. Во-первых, изучение факторов притяжения «Союза отцов» и «Совета отцов» к радикальным сообществам различного толка. Во-вторых, изучение процесса идеологической обработки в онлайн-среде членов сообществ ответственных отцов, чтобы понять, что заставляет некоторых из них присоединяться к крайне радикальным и даже экстремистским движениям и сообществам. В-третьих, изучение токсичного контента и возможностей цифровых эхо-камер радикальных сообществ, продвигающих идеи мизогинизма для понимания того, как такого рода идеи могут быть нормализованы и закреплены в риторике и дискурсе ответственных отцов. Соответственно, такого рода знание позволит сформировать превентивные меры на недопущение или предотвращение перехода в девиантные формы социальной активности. В-четвертых, необходимо расширение практики применения инструментов с использованием методов web mining, поскольку они позволяют получить результат в короткие сроки и обработать большой массив данных. Такая практика позволит исследователям создавать прогностические модели, чтобы оценить степень риска онлайн-радикализации.

Статья подготовлена при поддержке гранта Российского фонда фундаментальных исследований № 19-011-00644 «Генеративное поведение российскихмужчин вусловиях демографического кризиса: Приволжский и Северо-Западный округа РФ (сравнительный анализ)».

\section{СПИСОК ЛИТЕРАТУРЫ}

1. Ильдарханова Ч.И., Барсуков В.Н. Состояние и трансформация института отцовства: обзор зарубежных исследований // Гуманитарий Юга России. - 2019. - Т. 8. - № 6. - С. 211- 227.

2. Свидетельство «Генеративное поведение российскихмужчин в условиях демографического кризиса» // Академия наук республики Татарстан. URL: http://www.antat.ru/ru/csd/news/14075/ (дата обращения 03.09.2021).

3. Ильдарханова Ч.И. Габитус генеративного поведения молодых российских мужчин (опыт эмпирического исследования) // Государственная молодежная политика: национальныепроекты 2019- 2024 гг. в социальном развитии молодежи. - М.: Перспектива, 2020. - С. 97-103.

4. Давлетшина Л.А. Методологические основания анализа генеративного поведения мужчин. Ученые записки Крымского федерального университета имени В.И. Вернадского // Социология. Педагогика. Психология. - 2019. - № 1. - С. 51-55.

5. Горелик В.П. Некоторые соображения обисследовании генеративного поведения семьи // Демогр афические тетради. Киев. - 1972. - Вып. 4-5. - С. 24-27.

6. Давлетшина Л.А. Концептуализация генеративности семьи в аспекте реализации гражданского капитала: реляционный подход// Политическое пространство и социальное время:Глобальныевызовы и цивилизационные ответы: Сборник научныхтрудов XXXVII Международного Харакского форума. В 2 томах. - Симферополь: ИТ «АРИАЛ», 2021. - С. 235-241.

7. Калачикова О.Н., Груздева М.А. Детерминация моделей отцовства: экономический подход// Институты развития человеческого потенциала в условиях современных вызовов: Сборник статей XI Уральского демографического форума. В 2 томах. - Екатеринбург: Институт экономики Уральского отделения Российской Академии Наук, 2020. - С. 146-153.

8. Singley S.G. Transitions to parenthood: work-family policies, gender, and the couple context // Gender \& Society. - 2005. - V. 19. - Iss. 3. - P. 376-397. DOI: 10.1177/0891243204271515

9. Randles J. «Manning Up» to bea good father: hybrid fatherhood, masculinity, and U.S. responsible fatherhood policy // Gender \& Society. - 2018. - V. 32. - Iss. 4. - P. 516-539. DOI: https://doi.org/10.1177/0891243218770364

10. Closing the happiness gap: the decline of gendered parenthood norms and the increase in parental life satisfaction / K. Preisner, F. Neuberger, A. Bertogg, J.M. Schaub // Gender \& Society. - 2019. - V. 34. - Iss. 1. P. 31-55. DOI: https://doi.org/10.1177/0891243219869365 
11. Барсуков В.Н., Калачикова О.Н. Предпосылки и ограничения трансформации современного института отцовства // Социальное пространство. - 2021. - T. $7 . \quad$ - № 1. URL: http://sa.vscc.ac.ru/article/28858 (дата обращения 03.09.2021).

12. Социологическое исследование «Сетевые взаимодействия социально активных отцов: норма и о тклонения (анализ социальных сетей профильных НКО). Заявка № 2021621733 от 24.08.2021 // Ч.И. Ильдарханова, А.О. Савельев, А.Д. Вильнин, А.Ю. Карпова, А.Ю. Кайда, С.А. Кузнецов, A.M. Ширыкалов, Н.Г. Максимова.

13. Pro-life // Merriam-Webster. URL: https://www.merriam-webster.com/dictionary/pro-life (дата обращения 03.09.2021).

14. Munson Z. The making of pro-life activis ts: how social movement mobilization works. - Chicago: University of Chicago, 2009. - 248 p. DOI: 10.7208/chicago/9780226551210.001.0001

15. Hau geberg K. Women against abortion: inside the largest moral reform movement of the twentieth cent $u-$ ry. - Champaign, IL: University of Illinois Press, 2017. - 240 p. DOI: 10.5406/illinois/9780252040962.001.0001

16. Lithwick D. Poster children. After 9/11, what can abortion protesters get away with? URL: https://slate.com/news-and-politics/2002/05/after-9-11-what-can-abortion-protesters-get-away-with.html (дата обращения 03.09.2021).

17. Avast Academy. What is doxing and how can you prevent it? URL: https://www.avast.com/c-what-isdoxxing\#topic-1 (дата обращения 03.09.2021).

18. Meserve H.C. Editorial: pro-life, pro-choice // Journal of Religion and Health. - 1983. - № 22 (1). - P. 3-6.

19. Piper K. Pro-Life and pro-choice: what does it mean? Focus on the Family. October 2020. URL: https://www.focusonthefamily.com/pro-life/pro-life-pro-choice/ (дата обращения 03.09.2021).

20. Изучение процесса онлайн радикализации молодежи в социальных медиа (междисциплинарный подход) / А.Ю. Карпова, А.О. Савельев, А.Д. Вильнин, Д.В. Чайковский // Мониторинг общественного мнения: экономические и социальные перемены. - 2020. - № 3 (157). - С. 159-181.

21. Карпова А.Ю., Ширыкалов А.М. Разработка метода схожести коллекций текстов с использованием их векторныхпредставлений, полученныхметодом doc2vec// Молодежь и современные информационные технологии: Сборник трудов XVIII Международной научно-практической конференции студентов, аспирантов и молодыхученых. - Томск: Изд-во ТПУ, 2021. - С. 75-76.

Поступила 12.10.2021 2. 
UDC 316.624-055.52-055.1

\title{
TRANSFORMATION OF SOCIAL PRACTICES OF RESPONSIBLE FATHERHOOD INTO DEVIANT FORMS OF SOCIAL ACTIVITY
}

\author{
Anna Yu. Karpova, \\ belts@tpu.ru \\ Aleksey 0. Savelev, \\ sava@tpu.ru \\ Sergey A. Kuznetsov, \\ ksa11@tpu.ru \\ National Research Tomsk Polytechnic University, \\ 30, Lenin avenue, Tomsk, 634050, Russia
}

Anna Yu. Karpova, Dr. Sc., professor, National Research Tomsk Polytechnic University.

Aleksey 0. Savelev, Cand. Sc., associate professor, National Research Tomsk Polytechnic University.

Sergey A. Kuznetsov, postgraduate student, junior research fellow, National Research Tomsk Polytechnic University.

The study of social practices of responsible fatherhood is relevant at the present time in the context of the transformation of the institution of fatherhood. Particular attention of researchers is directed to the social construction of responsible fatherhood in the media space - the profile social networks of communities. The problem is that within fathers' communities in social networks there are markers of radical opinions, ideas, which are essentially predictors of the transition to deviant forms of social activity and destructive practices. The aim of the study is to assess the semantic similarity and cross-links between the communities of «Fathers' Union» and «Fathers' Council» and communities with signs of radicalization. Methods: content analysis and web mining. According to the results of the study the presence of a strong relation of thematic and semantic similarities between the communities of «fathers» and «pro-lifers» is indicated. There are signs of the transformation of social practices ofresponsible fatherhood into deviant forms of social activity. It is emphasized that the use of various methods of web mining increases the possibility to assess the influence of radical opinions andideas in the onlineenvironment, respectively, the possibility to prevent such form of social activity in the communities of responsible fathers as dissemination of radical ideology increases. In conclusion, future research directions are outlined.

Key words: Generative behavior, radicalization, extremism, network topology, big data, web mining.

The paper is supported by the grant of the Russian Foundation for Basic Research no. 19-011-00644 «generative behavior of Russian men within the demographic crisis: Volga and North-Western districts of the RF (comparative analysis)».

\section{REFERENCES}

1. Ildarkhanova Ch.I., Bars ukov V.N. Sos toyanie i transformatsiy ains tituta ottsovstva: obzor zarubezhnykh is sledovaniy [The state and transformation of the ins titution of paternity: a review of foreign studies]. Gumanitariy Yuga Rossii, 2019, vol. 8, no. 6, pp. 211-227.

2. Svidetelstvo «Generativnoe povedenie rossiyskikh muzhchin v usloviyakh demograficheskogo krizisa» [Testimony «Generative behavior of Russian men in the context of the demographic crisis»]. Akademiya nauk respubliki Tatarstan. A vailable at: http://www.antat.ru/ru/csd/news/14075/ (accessed 3 September 2021). 
3. Ildarkhanova Ch.I. Gabitus generativnogo povedeniya molodykh rossiyskikh muzhchin (opyt e mpiriches kogo is sledov aniya) [The habit of generative behavior of young Rus sian men (experience of empirical res earch)]. Gosudarstvennaya molodezhnaya politika: natsionalnye proekty2019-2024 gg. v sotsialnom razvitii molodezhi [State Youth Policy: National Projects 2019-2024 in the social development of youth]. Moscow, Perspektiva Publ., 2020. pp. 97-103.

4. Davletshina L.A. Metodologicheskie os novaniya analiza generativnogo povedeniya muzhchin. Uchenye zapiski Kryms kogo federalnogo universiteta imeni V.I. Vernadskogo [Metho dological foundations for the analysis of generative behavior in men. Scientific notes of the Crimean Federal University named after V.I. Vernadsky]. Sotsiologiya. Pedagogika. Psikhologiya, 2019, no. 1, pp. 51-55.

5. Gorelik V.P. Nekotorye soobrazheniya ob is sledovanii generativnogo povedeniy a semi [Some considerations about the study of generative family behavior]. Demograficheskie tetradi. Kiev, 1972, Is s. 4-5, pp. 24-27.

6. Davletshina L.A. Konts eptualizatsiya generativnosti semi v aspekte realizatsii grazhdan skogo kapitala: relyatsionny podkhod [Conceptualization of the generativity of the family in the aspect of the implementation of civil capital: a relational approach]. Sbornik nauchnykh trudov XXXVII Mezhdunarodnogo Kharakskogoforuma. Politicheskoe prostranstvo i sotsialnoe vremya: Globalnye vyzovyi tsivilizatsionnye otvety [Political s pace and social time: global challenges and civilizational responses. Collection of scientific papers of the XXXVII International Harak Forum. In 2 vol.]. Simferopol, ARIAL Publ., 2021. pp. $235-241$.

7. Kalachikova O.N., Gruzdeva M.A. Determinatsiy a modeley ottsovstva: ekonomicheskiy podkhod [Determination of models of paternity: an economic approach]. Sbornik statey XI Uralskogo demograficheskogo foruma. Instituty razvitiya chelovecheskogo potentsiala $v$ usloviyakh sovremennykh vyzovov [Institutions for the development of human potential in the face of modern challenges. Collection of articles of the XI Ural Demographic Forum. In 2 vol.]. Ekaterinburg, In stitut ekonomiki Urals kog o otdeleniy a Ros siyskoy Akademii Nauk Press, 2020. pp. 146-153.

8. Singley S.G. Transitions to parenthood: work-family policies, gender, and the couple context. Gender \& Society, 2005, vol. 19, Iss. 3, pp. 376-397. DOI: 10.1177/0891243204271515

9. Randles J. «Manning Up» to be good father: hybrid fatherhood, masculinity, and U.S. responsible fatherhood policy. Gender \& Society, 2018, vol. 32, Iss. 4, pp. 516-539. DOI: https://doi.org/10.1177/0891243218770364

10. Preisner K., Neuberger F., Bertogg A., Schaub J.M. Closing the happiness gap: the decline of gendered parenthood norms and the increase in parental life satisfaction. Gender \& Society, 2019, vol. 34, Iss. 1, pp. 31-55. DOI: https://doi.org/10.1177/0891243219869365

11. Barsukov V.N., Kalachikova O.N. Predposylki i ogranicheniya transformatsii sovremennogo instituta ottsovstva [Preconditions and limitations of the transformation of the modern institution of paternity]. Sotsialnoe prostranstvo, 2021, vol. 7, no. 1. Available at: http://sa.vscc.ac.ru/article/28858 (accessed 3 September 2021).

12. Ildarkhanova Ch.I., Savelyev A.O., Vilnin A.D., Karpova A.Yu., Kayda A.Yu., Kuznetsov S.A., Shirykalov A.M., Maksimova N.G. Sotsiologicheskoe issledovanie «Setevye vzaimodeystviya sotsialno aktivnykh ottsov: norma i otkloneniya (analiz sotsialnykh seteyprofilnykh NKO) » [Sociological res earch «Network interactions of socially active fathers: norm and deviations (analys is of social networks of specialized nonprofit organizations)»]. Svidetelstvo o registratsii bazy dannykh 2021621866, 03.09.2021. Zayavka no. 2021621733 ot 24.08 .202$.

13. Pro-life. Merriam-Webster. Available at:https://www.merriam-webster.com/dictionary/pro-life (acces sed 03 September 2021).

14. MunsonZ. The making ofpro-life activists: how socialmovementmobilization works. Chicago, University of Chicago, 2009. 248 p. DOI: 10.7208/chicago/9780226551210.001.0001

15. Haugeberg K. Women against abortion: inside the largestmoral reform movementof the twentieth century. Champaign, IL. University of Illinois Press, 2017. 240 p. DOI: 10.5406/illinois/9780252040962.001.0001

16. Lithwick D. Poster children. After 9/11, what can abortion protesters get away with? Available at: https://slate.com/news-and-politics/2002/05/after-9-11-what-can-abortion-protesters-get-away-with.html (accessed 3 September 2021).

17. Avast Academy. What is doxing and how can you prevent it? Available at: https://www.avast.com/c-whatis-doxxing\#topic-1 (acces sed 3 September 2021).

18. Meserve H.C. Editorial: pro-life, pro-choice. Journal of Religion and Health, 1983, no. 22 (1), pp. 3-6.

19. Piper K. Pro-Life and pro-choice: what does it mean? Focus on the Family. October 2020. Available at: https://www.focus on thefamily.com/pro-life/pro-life-pro-choice/ (accessed 3 September 2021).

20. Karpova A.Yu., Savelev A.O., Vilnin A.D., Chaykovskiy D.V. Izuchenie protsessa onlayn radikalizatsii molodezhiv sotsialnykh media (mezhdistsiplinarny podkhod) [Studying online radicalization of youth in 
social media (an interdis ciplinary approach)]. Monitoring obshchestvennogo mneniya: ekonomiche skiei sotsialnye peremeny, 2020, no. 3 (157), pp. 159-181.

21. Karpova A.Yu., Shirykalov A.M. Razrabotka metoda skhozhesti kollektsiy tekstov s is polzovaniyem ikh vektornykh predstavleniy, poluchennykh metodomdoc2vec [Development of a method for the similarity of text collections using their vector representations obtained by the doc2vec method]. Sbornik trudov XVIII Mezhdunarodnoy nauchno-prakticheskoykonferentsii studentov, aspirantovi molodykh uchenykh. Molodezh i sovremennye informatsionnye tekhnologii [Youth and Modern Information Technologies. Proceedings of the XVIII International Scientific and Practical Conference of Students, Postgraduates and Young Scie ntists]. Tomsk, TPU Publ., 2021. pp. 75-76.

Received: 12 October 2021. 